\title{
La inteligencia organizacional: necesario enfoque de gestión de información y del conocimiento
}

\author{
Yunier Rodriguez Cruz \\ Docente del Departamento de Bibliotecología y Ciencia de la Información de la \\ Facultad de Comunicación de la Universidad de La Habana \\ E-mail: yunier@fcom.uh.cu \\ Esther Galán Domínguez \\ Docente del Dep. de Bibliotecología y Ciencia de la Información de la Facultad de \\ Comunicación de la Universidad de La Habana. \\ E-mail: esthergalan@fcom.uh.cu
}

\begin{abstract}
RESUMEN
Se examina el comportamiento y desarrollo de la Inteligencia organizacional a partir del análisis de las concepciones que más han aportado a su evolución. Se examinan sus procesos: percepción, creación de conocimiento y toma de decisiones así como sus características fundamentales. Se presentan algunas consideraciones acerca de la importancia que presentan la información y el conocimiento y su adecuada gestión, en la inserción y desarrollo de la Inteligencia Organizacional en cualquier tipo de organización. La Inteligencia Organizacional posee un mayor alcance que las prácticas asociadas a la Inteligencia Competitiva, entre otras. Sin embargo, la integración de éstas permite generar dicha capacidad. Los procesos de la Inteligencia: percepción, creación de conocimiento y toma de decisiones tienen una marcada dependencia del uso y manejo de información y conocimiento. Todos esto permite asegurar que un adecuado y óptimo desarrollo de esta capacidad está condicionado por la aplicación de la Gestión de Información y del Conocimiento como procesos que garantizan beneficios del uso de estos recursos.
\end{abstract}

\section{PALABRAS CLAVE}

Inteligencia organizacional. Inteligencia empresarial. Gestión de información. Gestión del conocimiento.

\section{The organizational intelligence: a necessary approach to information and knowledge management}

\begin{abstract}
The current development of organizational intelligence is examined, starting from the analysis of the most important trends in its evolution. The characteristics of organizational processes such as perception, knowledge creation and decision making are revised. The author also presents some considerations about the relevance of information and knowledge, as well as their management, for the development of the organizational intelligence in any sort of institution. Organizational intelligence has a larger dimension than other practices associated to Competitive Intelligence. Nevertheless, the integration of those practices generates the organizational Intelligence. Intelligence processes depend remarkably on the use of information and knowledge. That way, the optimal development of organizational intelligence is affected by the application of Information and knowledge management.
\end{abstract}

\section{KEYWORDS}

Organizacional intelligence. Enterprise intelligence. Information management. Knowledge management.

\section{INTRODUCCIÓN}

En el contexto actual de las organizaciones, así como en la literatura especializada se observa un incremento de prácticas o experiencias asociadas a la inteligencia. Si bien el término es utilizado en las ciencias sociales como una capacidad que poseen y desarrollan los individuos para intervenir de forma ventajosa sobre la realidad, por medio del uso de su conocimiento; en el ámbito empresarial u organizacional la noción de la inteligencia se refiere a la capacidad que poseen las mismas para desarrollar procesos de tratamiento y uso de información y de conocimiento que faciliten una efectiva toma de decisiones, generando ventajas competitivas.

Esta capacidad, sus sistemas, procesos, productos y servicios se han visto sustancialmente favorecidos por los cambios que han tenido lugar a raíz del propio desarrollo organizacional. Las instituciones modernas están conscientes de la importancia que tiene su ambiente externo - cada vez más dinámico para su desarrollo, lo que ha posibilitado que las mismas establezcan vínculos y alianzas entre ellas a fin de enfrentar las variaciones que se producen en estos ambientes y aprovechar mejor las fortalezas que brindan dichas relaciones. Todo esto ha permitido que las organizaciones reconozcan al cambio como un factor de mejora, y al mismo tiempo el rol de la información, del conocimiento, de las nuevas tecnologías y de los propios procesos gerenciales. Estas nuevas características han propiciado que las organizaciones se apoyen en la inteligencia para detectar cambios que se producen en su ambiente externo y adaptarse a estos de forma óptima.

La inteligencia no sólo tiene en cuenta a los cambios que se producen en su ambiente externo, sino también a los que tienen lugar dentro de la organización, es decir, su ambiente interno. En este sentido, es necesario aclarar que dada la relación de dependencia entre la institución y su ambiente externo, las variaciones o cambios que se produzcan en uno van a influir en el otro, de ahí que se deba reconocer la importancia de ambos y su relación para el desarrollo de esta capacidad.

Inteligencia Organizacional $(\mathrm{IO})^{1}$

\footnotetext{
1 En la literatura especializada se usan indistintamente los términos Inteligencia Empresarial,
} Corporativa u Organizacional, para definir esta capacidad. 
Entre las conceptualizaciones del término se reconocen autores como Wilensky (1967) y Aeckel y Nolan (1993). Sus criterios apuntan a tres elementos importantes: (1) la información proveniente del ambiente externo y su uso; (2) los procesos informacionales para su adecuado tratamiento (búsqueda, selección, procesamiento, análisis y diseminación) y (3) la toma de decisiones como proceso estratégico para adaptarse a su ambiente externo o mercado.

Las concepciones actuales, por su parte, muestran la evolución conceptual del tema en correspondencia con las nuevas características y exigencias de las organizaciones. Autores como Choo, Halal, McMaster, Cubillo y Orozco, valoran la IO como una capacidad organizacional e incorporan como elementos fundamentales: (1) el conocimiento y los procesos asociados a este recurso; (2) la toma de decisiones para la orientación estratégica y (3) el aprendizaje organizacional.

En esencia, la IO constituye una capacidad organizacional, desarrollada por medio del aprendizaje sistemático, que permite a la organización percibir adecuadamente su ambiente externo e interno por medio del uso y procesamiento de la información proveniente de estos, y generar nuevos conocimientos organizacionales que contribuyan a una efectiva toma de decisiones para la resolución de problemas y la orientación estratégica ante ambientes cada vez más cambiantes.

\section{¿Organizaciones Inteligentes?}

Algunos autores plantean que una organización alcanza esta condición en dependencia del nivel de inteligencia que posean sus especialistas y otros lo atribuyen a la información y al uso de este recurso en la organización.

El primer criterio se sustenta en el hecho de que son los individuos los que perciben las características del ambiente externo e interno de la organización, es decir, identifican, analizan, interpretan y comprenden los cambios, y a su vez, son los que crean nuevos conocimiento en busca de una solución efectiva para adaptarse a los mismos e implementan las acciones para ello. El segundo se sustenta en la importancia de ese recurso para el desarrollo de la inteligencia ya que si no se dispone de la información necesaria no se podría conocer y comprender los cambios que afectan a la organización.

En muchas ocasiones estamos en presencia de organizaciones que cuentan con especialistas muy preparados e inteligentes, y sin embargo, no logran comprender adecuadamente su ambiente externo e interno, ni generar ideas novedosas en la solución de problemas o encontramos organizaciones que disponen de la información necesaria y sin embargo no le brindan un tratamiento y uso adecuado. Esto ocurre al no valorar la interdependencia de estos recursos, por lo que se puede afirmar que la información, los individuos y su conocimiento, constituyen recursos de vital importancia para el de desarrollo de la IO.

\section{Figura 1}

Relación entre Individuos y/o Grupo Socioal-Información-

Conocimiento-Toma de Decisiones

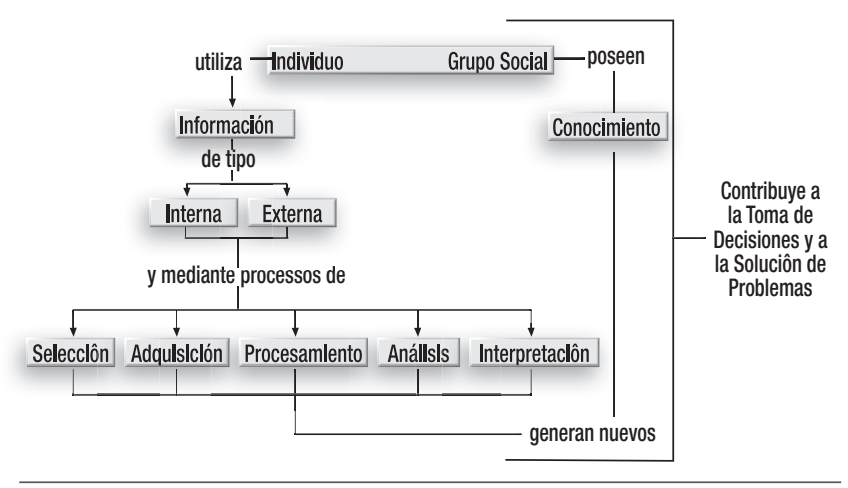

Fuente: Elaboración propia

Todo esto permite afirmar que los factores críticos de éxito para la inteligencia lo constituye la relación de dependencia que se establece entre: (1) los individuos y grupos o redes sociales que estos conforman; (2) la información y (3) el conocimiento.

Dicha relación y su importancia se evidencia en las definiciones de organizaciones inteligentes de autores como Halal, Choo, Garvin, Senge y Nuñez. Nuñez (2002) plantea que una

organización inteligente (que posee la capacidad de la inteligencia) es una organización de aprendizaje, que promueve la socialización e incorporación de conocimientos a fin de mejorar las capacidades creativas de las personas, unificar los objetivos y los significados de la comunidad que la integra, mediante la conciencia y la capacidad para el trabajo en equipo y una percepción y razonamiento integral de todos los sistemas que intervienen en la vida de la organización.

Choo (1999) plantea que la organización que sea capaz de

integrar eficazmente la percepción, la creación de conocimiento y la toma de decisiones se puede describir como una organización inteligente: posee información y conocimiento, por lo que está bien informada, es mentalmente perceptiva y clara. Sus acciones se basan en una comprensión compartida y válida del medio ambiente y las necesidades de la organización, y son influidas por los recursos de conocimiento disponibles y competencias en cuanto a habilidades de sus miembros.

Una organización inteligente es aquella que prevé, se anticipa y adapta a los cambios de su entorno, ya que conoce y comprende las características de ese medio en el que se desenvuelve y es capaz de movilizar todo su conocimiento para, por medio de la innovación, resolver de forma rápida y creativa los problemas que se le presenten, actuando de forma inteligente. 


\section{PRINCIPALES CARACTERÍSTICAS DE LA IO}

La IO, a consideración de Choo (1999), posee las siguientes características: (1) mediada, (2) situada, (3) provisional, (4) pragmática y (5) disputada.

La primera hace alusión a que la inteligencia se encuentra condicionada por los sistemas, es decir, los individuos que los conforman y la interacción que los mismos establecen con fuentes de información, nuevas tecnologías y otros recursos materiales para el desarrollo de los procesos.

La segunda localiza a la inteligencia bajo las condiciones espacio-temporales, ya que la misma está sujeta a la realidad existente y circundante en la cual se desenvuelve la organización.

Es provisional dado que depende en gran medida de las rutinas, reglas y políticas bajo las que se desempeña la institución, que al mismo tiempo están sujetas a variaciones.

El pragmatismo viene dado porque produce una acción enfocada a fines organizacionales y dirigida al objeto de la actividad, y dicha acción depende de las concepciones que posean los especialistas.

Por último, su carácter disputado remite a los conflictos que pueden generarse a raíz del desarrollo de la toma de decisiones, el acceso a la información etc.

\section{Procesos que intervienen en la IO}

En una organización, el desarrollo de la IO se lleva a cabo en la medida en que se logren ejecutar los procesos asociados a la información, al conocimiento y a las decisiones. En el "Ciclo de Vida de la Inteligencia" en las organizaciones, presentado por Choo (1999), se identifica la interrelación de los tres procesos fundamentales que a consideración del autor conforma esta capacidad (IO): Percepción, Creación de Conocimiento y Toma de Decisiones. Cada uno de estos procesos se efectúa a partir del uso de la información, por lo que este autor considera que constituyen procesos informacionales.

\section{La Percepción Organizacional}

La percepción permite a la organización representar su ambiente externo al identificar las características que presenta, de igual forma contribuye a determinar las fortalezas y debilidades que ésta posee y conformar o modificar su propia identidad.

La percepción presenta, según Weick (1995), siete propiedades, entre las cuales encontramos su carácter social, constante y retrospectivo ya que la misma se lleva a cabo por varios individuos, es un proceso continuo y constante de aprendizaje y tiene en cuenta situaciones y acontecimientos que han sucedido en la institución. Este proceso se basa en la construcción de la identidad de las organizaciones y tiende a reproducir el ambiente en el que ésta se desenvuelve. Otros elementos importantes son el hecho que se concentra en indicaciones extraídas o información que permite conocer lo que ocurre y es impulsada por la plausibilidad antes que por la exactitud.

El propio autor señala que la percepción se logra a partir de la interrelación de los siguientes procesos: (1) Variación Ecológica, (2) Representación, (3) Selección y (4) Retención.

La variación ecológica tiene lugar cuando se detecta un cambio en el ambiente externo y/o interno de la organización, el que se necesita identificar y comprender adecuadamente. La representación se efectúa cuando los miembros de la institución determinan las características de este y valoran qué incidencias tiene o puede tener sobre la organización. En este proceso se deben obtener datos que sean completos, precisos y confiables para evitar informaciones y análisis erróneos. En la selección se buscan respuestas a la realidad que acontece interpretándose los cambios por medio de la información recopilada y determinando posibles causas, por lo que no sólo se analiza la información sino que se valoran las experiencias y la forma de tratar situaciones de este tipo en la organización. El último proceso pretende documentar y almacenar los resultados de una percepción efectiva para que se puedan recuperar en un futuro y contribuir de esta manera al enriquecimiento de la memoria organizacional.

En la percepción organizacional el proceso fundamental es la interpretación del ambiente ya que esta es la que facilita una adecuada representación y caracterización del mismo. Este proceso, que conlleva al análisis de la información asociada a los cambios, se debe realizar de forma colectiva entre todos los miembros de la organización, ya que cada uno de ellos, en un principio, realizan su propia interpretación de la situación, y es necesario el intercambio y la reflexión colectiva de los mismos para obtener como producto final una única representación del ambiente, compartida por todos.

\section{La Creación de Conocimiento Organizacional}

La Creación de Conocimiento Organizacional, según Nonaka y Takeuchi (1995),

debe entenderse como la capacidad orgánica para generar nuevos conocimientos, difundiéndolos entre los miembros de una organización y materializándolos en productos, servicios y sistemas. Es la clave del proceso a través del cual las firmas innovan

Según McElroy (2000),

la producción de conocimientos en una organización es un proceso socialmente emergente. Los sistemas sociales por su naturaleza intrínseca dan lugar a una elaboración 
colectiva del conocimiento por parte de sus miembros, como un producto derivado de su aprendizaje individual y su interacción interpersonal.

González (2001), por su parte, reconoce como proceso de creación de conocimiento a la:

(1) Generación de ideas: se crea nuevo conocimiento; (2) Evaluación de ideas generadas: las ideas son evaluadas en función de su utilidad y su eficiencia para mejorar las tareas; (3) Transferencia de ideas evaluadas: las ideas que son apropiadas se transfieren a todas las áreas de la empresa y (4) Asimilación de las ideas transferidas: las ideas son retenidas y adquieren la forma de rutinas organizacionales.

El resultado de este proceso son un conjunto de conocimiento que circulan por los diferentes niveles organizacionales de conocimiento: individual, grupal y organizacional. Según las consideraciones de autores como Polanyi, Nonaka y Takeuchi, Brown y Gray, McElroy y Choo acerca de los tipos de conocimiento se pueden identificar en cada nivel la presencia del conocimiento tácito y el explícito.

El conocimiento tácito es

aquel que forma parte de las experiencias de aprendizaje personal de cada individuo y que por tanto resulta sumamente complicado, imposible de estructurar, almacenar en repositorios y distribuir

Por su parte el conocimiento explícito es aquel que se puede expresar con palabras y números, y comunicarse y compartirse con facilidad en la forma de datos concretos y fórmulas científicas, procedimientos codificados, o principios universales.

Estos conocimientos son los que apoyan el proceso de la innovación, pero la creación de los mismos está condicionada por las formas de creación y conversión que adopte cada organización. Entre los modelos de creación de conocimiento se encuentran el de Nonaka y Takeuchi (1995) y el de Carrión (2002). El primero muestra la creación o conversión de conocimiento por medio de los procesos de Socialización, Externalización, Internalización y Combinación, y el segundo muestra la dinámica de creación a partir de las redes sociales y del uso de nuevas tecnologías, así como las ventajes que brinda el conocimiento una vez creado.

En cuanto a los procesos de creación mencionados anteriormente, es necesario plantear que en el Modelo de Crítica desarrollado por García se analiza cómo estos procesos deben desarrollarse no sólo en cada nivel de conocimiento, sino que debe ser la vía para generar conocimientos de un nivel a otro, logrando de esa forma la creación de conocimiento organizacional.

La importancia de este proceso radica en las ventajas que brinda el conocimiento para la toma de decisiones y la solución de problemas en la organización de forma efectiva. La única vía para que las instituciones logren generar ideas novedosas y la forma de llevarlas acabo es mediante la creación y uso del conocimiento.

\section{La Toma de Decisiones en la organización}

La toma de decisiones tiene lugar en todas las organizaciones y se basa en la elección de una alternativa para resolver un problema determinado que en esta tenga lugar. Si bien la decisión es la que permite realizar soluciones, lo importante radica en el proceso que realiza la organización para tomarla, ya que es este el que asegura la efectividad de la misma.

Schein identifica a la toma de decisiones como "el proceso de identificación de un problema u oportunidad y selección de una alternativa de acción entre varias existentes, constituyendo una actividad diligente clave en todo tipo de organización".

La toma de decisión tiene como componentes fundamentales a: (1) Situación problémica: determina el desarrollo del proceso y que el mismo tenga lugar; (2) Individuo: posibilita el razonamiento en la toma de decisión mediante el análisis de posibles alternativas y (3) Información derivada del problema: permite asegurar la objetividad del análisis que se realiza, de ahí que la información y los recursos para su uso y tratamiento sean fundamentales en este proceso.

Este proceso es importante para la $\mathrm{IO}$ ya que permite la elección de una alternativa de solución y el mecanismo o las vías para llevarla a cabo. Esta alternativa es precisamente la que permite la adaptabilidad de la organización ante los cambios que se producen en su ambiente externo e interno, de ahí que el proceso en sí sea decisivo para el logro de los objetivos de la inteligencia.

\section{LA INFORMACIÓN EN LA INTELIGENCIA ORGANIZACIONAL}

La información juega un papel fundamental en la Inteligencia Organizacional. Este recurso, que no es más que un conjunto de datos relacionados significativamente que modifica o transforma el conocimiento que posee un individuo de acuerdo al contexto en el que esta surge y se desenvuelve, es el que permite a la organización identificar, tratar y adaptarse a los cambios externos e internos que se le presentan.

En las organizaciones la noción o concepciones de la información se sintetizan según Braman en los siguientes aspectos: la información como recurso, como activo, como percepción de patrón y como fuerza constitutiva en la sociedad. La Inteligencia Organizacional tiene que reconocer cada uno de ellos dado su alcance de capacidad organizacional, lo que hace que su interés por la información abarque todas las dimensiones asociadas a ella en la organización. Su noción 
como recurso permite valorarla como entidades que entran y circulan por la organización en pedazos inconexos o flujos de información en los cuales podría estar organizada. La información como activo incorpora el papel que esta juega en la relación que se establece entre los individuos y las actividades organizacionales validando la potencia económica de la información al mostrar lo que implica su uso.

La percepción de patrón alude a su carácter contextual ya que esta se afecta por determinados factores ambientales y causales y a su vez tiene efectos en sí misma y su noción de fuerza constitutiva parte de que esta no es afectada por su ambiente pero es en sí misma un agente que incide en otros elementos de este.

La importancia de la información en la Inteligencia Organizacional radica fundamentalmente en que este recurso está presente en cada proceso asociado a esta capacidad.

\section{En la percepción}

La información constituye un elemento imprescindible en el proceso de percepción organizacional. El uso de este recurso está presente en el desarrollo de los procesos que lo conforman:

Variación Ecológica: la identificación de las situaciones problémicas y de los cambios se realiza a través de las señales que se reciben de los ambientes externos o internos. Estas señales no son más que informaciones que se obtienen directa e indirectamente a través de fuentes de información documentales y no documentales. En este proceso es necesario validar esas fuentes para garantizar la veracidad de la información.

Representación: se obtienen todos los datos posibles que permitan caracterizar los cambios y la realidad del ambiente. En este proceso es preciso tener en cuenta que la información necesita ser lo más completa, veraz y oportuna para evitar inadecuadas representaciones del ambiente. En este sentido la búsqueda y selección de información es muy importante, por ende es importante establecer de forma consecuente mecanismos relacionados al monitoreo del ambiente, identificación y análisis de fuentes de información así como un acertado procesamiento y almacenamiento de la información recopilada.

Selección: en este proceso, en el cual se desarrolla la interpretación, el análisis de la información es fundamental, ya que es el que permite conocer el origen de los cambios, las causas que los provocan y las consecuencias que estos traen consigo.

Retención: en este proceso se documentan las experiencias de cada proceso de percepción y se almacenan para una futura recuperación. Esta información que se genera permite a la organización no solo ahorrar tiempo, sino facilitar las percepciones futuras.

\section{En la creación de conocimiento}

En la creación o transformación de conocimientos, la información es importante ya que esta constituye la expresión del mismo. Los procesos de transformación de conocimiento - dígase socialización, externalización, internalización y combinación - son proceso informacionales por el uso que hacen de este recurso.

Socialización: los individuos o grupos comparten sus experiencias o conocimientos mediante diversas vías - entre las que sobresalen la observación y el diálogo - que llevan explícito el intercambio de información.

Externalización: se expresan o se documentan los conocimientos que un individuo posee, es decir se explícita ese conocimiento en información.

Combinación: permite crear conocimiento a partir del uso y análisis de información, ya que los individuos interpretan o analizan información que puede o no estar documentada y luego de hacerlo generan un nuevo conocimiento.

Internalización: en este proceso se aprende e interiorizan nuevos conocimientos a partir del análisis de información.

En cada uno de estos procesos la información es imprescindible, en ocasiones esta se presenta mediante palabras, gestos o documentos y contribuye a que los individuos creen sus conocimientos.

\section{En la toma de decisiones}

La información en la toma de decisiones asegura la objetividad del análisis que se realiza de ahí que su uso y tratamiento sean imprescindibles para garantizar la selección con la racionalidad requerida de una o varias alternativas efectivas en el momento de tomar una decisión, por lo que no solo debe ser confiable, completa y oportuna sino que debe validarse y procesarse a fin de que disminuya la incertidumbre en el análisis.

Choo (1999) plantea que en la toma de decisiones es necesario conocer información sobre el estado actual (de qué alternativas se dispone), información sobre el futuro (consecuencias que generaría cada una) e información asociada a las acciones, para llevar a cabo las alternativas que se tomen y lograr los beneficios de esta.

\section{LA GESTIÓN DE INFORMACIÓN EN LA INTELIGENCIA ORGANIZACIONAL}

La Gestión de Información contribuye a la eficacia, eficiencia y productividad de las organizaciones y permite que todos sus miembros dispongan de forma adecuada de los recursos 
informativos que existen en ella, así como de aquellos que necesita, lo que les permite insertarse, con la calidad requerida, en los procesos y proyectos que desarrolla la organización.

La Gestión de Información, según Ponjuán (1998) es el

proceso mediante el cual se obtienen, despliegan o utilizan recursos básicos (económicos, físicos, humanos, materiales) para manejar información dentro y para la sociedad a la que sirve. Tiene como elemento básico la gestión del ciclo de vida de este recurso y se desarrolla en cualquier organización.

Bustelo (2001) define la Gestión de Información como el

conjunto de actividades realizadas con el fin de controlar, almacenar y, posteriormente, recuperar adecuadamente la información producida, recibida o retenida por cualquier organización en el desarrollo de sus actividades.

La Gestión de Información constituye un proceso mediante el cual se planifican, organizan, dirigen y controlan los recursos de información de una organización asegurando un adecuado tratamiento, intercambio y uso de este recurso, que contribuyan al establecimiento de fortalezas organizacionales. Al desarrollar la Gestión de Información la organización logra identificar y adquirir la información necesaria para satisfacer sus necesidades informativas y organizar dicha información para un acceso cómodo y rápido.

\section{El conocimiento en la Inteligencia Organizacional}

El conocimiento es un recurso de gran importancia para la IO. Davenport y Prusack (1995) plantean que el conocimiento es una

mezcla fluida de experiencia, valores, información contextual y perspicacia del experto conectados entre sí que proveen un marco para la evaluación e incorporación de nuevas experiencias e información. Este es originario y aplicado en las mentes de los conocedores. En los organismos, el conocimiento comienza a ser frecuentemente incorporado no sólo en documentos y en sus depósitos, sino también en las rutinas organizacionales, prácticas y normas

y Páez Urdaneta (1992) en su pirámide informacional lo define como "estructuras informacionales que, al internalizarse, se integran a sistemas de relacionamiento simbólico de más alto nivel y permanencia". Es decir, el conocimiento se genera y se aplica en las mentes de los individuos una vez que se analizan, comprenden e interiorizan estructuras informacionales que pasan a formar parte del flujo de experiencia, valores y habilidades que estos poseen.

En las personas, este conocimiento se evidencia de dos formas, lo que un individuo sabe (knowwhat) y lo que sabe hacer (knowhow), por lo que ese conocimiento que a nivel teórico en ocasiones divisamos en las personas, se encuentra estrechamente relacionado con las habilidades que las mismas poseen debido a la propia dinámica de creación y uso de conocimiento, ya que al aprender algo nuevo se modifica o transforma ese conjunto de habilidades o formas de hacer que tiene un individuo.

En el contexto de las organizaciones el conocimiento tácito y explícito se incorpora en documentos, rutinas organizacionales, prácticas y normas. Esto no sucede siempre de forma aislada, dado que las organizaciones están conformadas por grupos o redes sociales. Esto hace que la creación, intercambio y uso de conocimiento se desarrolle a nivel individual, grupal y organizacional. El primer nivel responde al aprendizaje que tiene lugar en una persona y el modo en que esta crea conocimiento. El segundo ocurre cuando se conforman grupos de acuerdo a determinados intereses comunes dentro de la organización que por medio del aprendizaje grupal generan y asimilan nuevos conocimientos. En el tercero, el aprendizaje se realiza entre varios grupos y personas que por objetivos e intereses colectivos necesitan crear e intercambiar nuevos conocimiento.

\section{En la percepción}

Aún cuando los individuos realizan constantemente su propia percepción sobre determinados fenómenos de la realidad, en las organizaciones, el adecuado desarrollo de este proceso viene dado por el carácter social que el mismo presenta, lo que trae consigo que en el proceso de percepción el conocimiento que posee cada individuo sea imprescindible para entender y comprender el ambiente externo e interno, así como los conocimientos que se generan entre todos al percibirlos.

En la Variación Ecológica y la Representación, el conocimiento juega un papel fundamental ya que dependen de las habilidades y experiencias individuales, grupales y organizacionales que se tengan para identificar los cambios. Las experiencias que adquieren los miembros de la organización en percepciones realizadas con anterioridad ayudan a crear habilidades al identificar cuándo nos encontramos en presencia de un cambio y cuándo no, y crean habilidades para determinar necesidades informativas sobre estos y para validar fuentes de información que aporten información veraz, precisa y lo más completa posible.

En la selección, el conocimiento es imprescindible porque es el que permite interpretar los cambios, las causas que los originaron y las consecuencias que pueden ocasionar. Por su parte en la Retensión tiene gran importancia ya que en ella se explicitan los conocimientos derivados de la percepción, facilitando futuras percepciones organizacionales.

En general, la percepción depende de los modelos mentales. Una adecuada percepción es aquella que involucra todo el conocimiento organizacional que existe en la institución ya 
La inteligencia organizacional: necesario enfoque de gestión de información y del conocimiento.

que el resultado de la percepción no sólo nutre a los individuos de nuevos conocimientos y experiencias sino que los prepara y les permiten adquirir habilidades para tratar adecuadamente situaciones problémicas asociadas a los cambios.

\section{En la toma de decisiones}

En la toma de decisiones, este recurso es fundamental porque las alternativas de solución las generan los miembros de la organización atendiendo al conocimiento que poseen. Las decisiones se toman en dependencia de las experiencias y las rutinas y normas organizacionales, que en ocasiones pueden verse afectadas por la propia solución.

A la hora de elegir una alternativa se deben conocer las fortalezas y debilidades de la organización y el papel de la innovación como agente de cambio interno. Este proceso de innovación tributa a su vez al desarrollo organizacional.

\section{LA GESTIÓN DEL CONOCIMIENTO EN LA INTELIGENCIA ORGANIZACIONAL}

\section{Según Wiig (1997) la Gestión del Conocimiento}

se centra en facilitar y gestionar aquellas actividades relacionadas con el conocimiento, tales como su creación, captura, transformación y uso. Su función es la de planificar, poner en práctica, operar, dirigir y controlar todas las actividades relacionadas con el conocimiento y programas que se requieren para la gestión efectiva del capital estructural.

Ponjuán (2003) expresa que por Gestión del Conocimiento

se entiende el proceso sistemático e integrador, de coordinación de las actividades de adquisición, creación, almacenaje y comunicación del conocimiento tácito y explícito por individuos y grupos con objeto de ser más efectivos y productivos en su trabajo, y cumplir los objetivos y metas de la organización.

De acuerdo con estos conceptos, la Gestión del Conocimiento es un proceso que garantiza y facilita la creación, intercambio y uso de conocimiento al desarrollar acciones que permitan identificar, adquirir, almacenar, intercambiar, aplicar y asegurar el conocimiento en una organización e involucrar este recurso en la innovación y en el logro de altos indicadores de éxito y de mejoras continuas.

\section{La Inteligencia Organizacional como modelo integrado de Gestión de Información y del Conocimiento}

Como se ha demostrado la Inteligencia Organizacional depende de la información, el conocimiento y el uso que se proporciona a estos recursos en las organizaciones, por lo que la Gestión de Información y del Conocimiento influyen en el óptimo desarrollo de esta capacidad al crear las condiciones para tratar adecuadamente a los mismos.

\begin{tabular}{|c|c|}
\hline Gestión de Información. & $\begin{array}{l}\text { Inteligencia } \\
\text { Organizacional. }\end{array}$ \\
\hline $\begin{array}{l}\text { Permite una adecuada planificación, } \\
\text { organización, dirección y control de los } \\
\text { recursos informativos en la organización. }\end{array}$ & \multirow{12}{*}{$\begin{array}{l}\text { Los procesos de } \\
\text { percepción, creación } \\
\text { de conocimiento y } \\
\text { toma de decisiones } \\
\text { se ven favorecidos } \\
\text { ya que disponen } \\
\text { de una adecuada } \\
\text { gestión de los } \\
\text { recursos informativos } \\
\text { y por ende se } \\
\text { logra un mejor } \\
\text { uso y tratamiento } \\
\text { de la información } \\
\text { necesaria para su } \\
\text { desarrollo. }\end{array}$} \\
\hline $\begin{array}{l}\text { Monitorea sistemáticamente las necesidades } \\
\text { informativas de los miembros de la } \\
\text { organización. }\end{array}$ & \\
\hline $\begin{array}{l}\text { Coordina y/o desarrolla la ejecución } \\
\text { efectiva de procesos informacionales en la } \\
\text { organización. }\end{array}$ & \\
\hline $\begin{array}{l}\text { Integra los diversos sistemas informativos en } \\
\text { la organización para un óptimo uso de este } \\
\text { recurso. }\end{array}$ & \\
\hline $\begin{array}{l}\text { Utiliza plataformas o softwares que } \\
\text { contribuyan al intercambio y uso de la } \\
\text { información. }\end{array}$ & \\
\hline Beneficios & \\
\hline $\begin{array}{l}\text { Apoya el desarrollo de las funciones, } \\
\text { actividades y procesos de la organización. }\end{array}$ & \\
\hline $\begin{array}{l}\text { Facilita el desarrollo de procesos con una } \\
\text { marcada dependencia de la información. }\end{array}$ & \\
\hline $\begin{array}{l}\text { Garantiza el flujo constante de información en } \\
\text { la organización. }\end{array}$ & \\
\hline $\begin{array}{l}\text { Maximiza beneficios del uso de información } \\
\text { en la organización. }\end{array}$ & \\
\hline $\begin{array}{l}\text { Minimiza costos asociados a la información en } \\
\text { la organización. }\end{array}$ & \\
\hline $\begin{array}{l}\text { Facilita y optimiza la innovación ya que apoya } \\
\text { la Gestión del Conocimiento. }\end{array}$ & \\
\hline Gestión del Conocimiento. & $\begin{array}{l}\text { Inteligencia } \\
\text { Organizacional. }\end{array}$ \\
\hline $\begin{array}{l}\text { Permite una adecuada planificación, } \\
\text { organización, dirección y control de los } \\
\text { recursos organizacionales que permiten } \\
\text { desarrollar los procesos de conocimiento. }\end{array}$ & \multirow{11}{*}{$\begin{array}{l}\text { Los procesos de } \\
\text { percepción, creación } \\
\text { de conocimiento y } \\
\text { toma de decisiones } \\
\text { se ven favorecidos } \\
\text { en la medida en } \\
\text { que se optimizan } \\
\text { los procesos de } \\
\text { conocimiento, lo que } \\
\text { trae consigo que se } \\
\text { pueda disponer de } \\
\text { este recurso y su uso } \\
\text { en cada proceso de } \\
\text { la inteligencia. }\end{array}$} \\
\hline $\begin{array}{l}\text { Coordina y/o desarrolla los procesos } \\
\text { de conocimiento que tiene lugar en la } \\
\text { organización. }\end{array}$ & \\
\hline Producción de Conocimiento. (Identificación de & \\
\hline $\begin{array}{l}\text { conocimiento, identificación de requerimientos } \\
\text { de conocimiento, adquisición de conocimiento, } \\
\text { creación y validación de conocimiento) }\end{array}$ & \\
\hline $\begin{array}{l}\text { Integración de Conocimiento (Compartir, } \\
\text { Enseñar, Difundir, Proteger) }\end{array}$ & \\
\hline Beneficios & \\
\hline $\begin{array}{l}\text { Apoya el desarrollo de las funciones, } \\
\text { actividades y procesos de la organización. }\end{array}$ & \\
\hline $\begin{array}{l}\text { Facilita el desarrollo de procesos con una } \\
\text { marcada dependencia del conocimiento. }\end{array}$ & \\
\hline $\begin{array}{l}\text { Permite un mayor intercambio y difusión de } \\
\text { conocimiento. }\end{array}$ & \\
\hline $\begin{array}{l}\text { Garantiza un mayor aprovechamiento del } \\
\text { conocimiento como activo organizacional. }\end{array}$ & \\
\hline $\begin{array}{l}\text { Apoya el desarrollo de las funciones, } \\
\text { actividades y procesos de la organización por } \\
\text { medio del uso del conocimiento. }\end{array}$ & \\
\hline
\end{tabular}




\section{Figura 2}

Integración Gestión de Informació-Gestión del ConocimientoInteligencia Organizacional

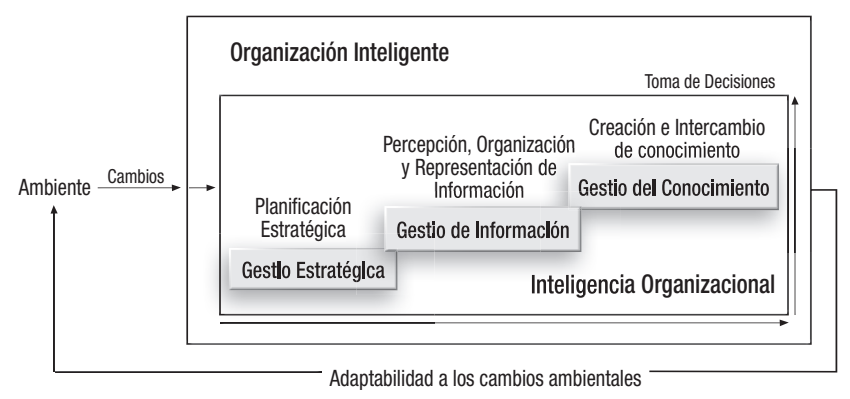

Fuente: Elaboración propia

La importancia e interrelación de la Gestión de Información y del Conocimiento en la IO nos permite afirmar que la implementación de la misma como capacidad está sustentada en el desarrollo de ambos.

La integración mostrada anteriormente es la que propicia que la organización pueda efectuar una toma de decisiones efectiva y que se adapte a los cambios de su ambiente.

\section{CONCLUSIONES}

- La IO constituye una capacidad organizacional que no responde a la suma de inteligencias de los miembros de una organización sino a la capacidad que tiene la misma de emprender acciones inteligentes.

- La IO posee un mayor alcance que otras prácticas asociadas al tema, aunque estas últimas tributan sin lugar a dudas al logro y óptimo funcionamiento de esta capacidad.

- La IO desarrolla procesos que le permiten a la organización detectar y comprender los cambios, crear conocimiento organizacional y tomar decisiones con la racionalidad requerida. Estos procesos presentan una marcada dependencia de la información y del conocimiento.

- La Gestión de Información permite a la organización administrar eficiente y eficazmente los recursos informativos por lo que contribuye a un mejor desarrollo de cada proceso de la IO.

- La Gestión del Conocimiento permite a la organización un adecuado uso de sus recursos para desarrollar los procesos de conocimiento por lo que contribuye a un óptimo desarrollo de cada proceso de la Inteligencia.

- La implementación de la IO como capacidad está sustentado en el desarrollo de la Gestión de Información y del Conocimiento, lo que permite crear las condiciones organizacionales para un acertado uso de estos recursos.

Artigo recebido em 10/04/2007

e aceito para publicação em 16/05/2008

\section{REFERÊNCIAS}

ARIÑO, M. A. Toma de decisiones y gobierno de organizaciones. Barcelona: Ediciones Deusto, 2005. 233 p.

BUSTELO, C.; AMARILLA, R. Gestión del conocimiento y gestión de la información. Boletín del Instituto Andaluz de Patrimonio Histórico, v. VIII, n. 34, p. 226-230, 2001. Disponível em: < $<$ http://www.inforarea.es/Documentos/GC.pdf $>$. Acesso em: 12 jan. 2006.

CASTELLS, P. Escorsa. De la vigilancia tecnológica a la inteligencia competitiva en las empresas. Disponível em: <http://www.uoc.es/web/esp/art/uoc/escorsa0202/escorsa 0202 imp.html >. Acesso: 05 maio 2006.

$\mathrm{CHOO}$, Chun Wei. Information management for the intelligent organization: the art of scanning the enviroment. New Jersey: American Society for the Information Science and Tecnology, 2002. $325 \mathrm{p}$.

La organización inteligente: el empleo de la información para dar significado, crear conocimiento y tomar decisiones. Cuauthémoc: Oxford University Pres, 1999. 288 p.

CUBILLO, J. La inteligencia empresarial en las pequeñas y medianas empresas competitivas de América Latina: algunas reflexiones. Ciência da Informação, v. 26, n. 3, p. 260-267, 1997. Disponível em: < http://www.scielo.br/ scielo.php?script $=$ sci_arttext\&pid $=$ S01009651997000300005 > . Acesso em: 10 out. 2005.

DAVENPORT, T. U.; PRUSAK, L. Conocimiento en acción: cómo las organizaciones manejan lo que saben. Buenos Aires: Pearson Education, 2001. 256 p.

DICK, B.; DALMAU, T. Argyris and schön: some elements of their models. Disponível em: < http://www.scu.edu.au/schools/gcm/ar/as/argyris2.html $>$. Acesso em: 15 set. 2005 .

GIFFORD; PINCHOT, E. The Intelligent organization. Disponível em: <http:// www.pinchot.com/MainPages/BooksArticles/IntelligentOrganization/TheIntellOrg. html>. Acesso em: 14 out. 2006.

HALAL, J. E. Organizational intelligence. Disponível em: < http://www.strategybusiness.com/press/article/12644?pg=0 > . Acesso em: 10 dez. 2005.

LARA, J. 1. Diez respuestas a las preguntas más frecuentes sobre gestión del conocimiento. Disponível em: < http://www.gestiopolis.com/canales/gerencial/Artículos 41/faqkm. htm>. Acesso em: 26 dez. 2005.

MACMASTER, M. Organizacional intelligence. Disponível em: < http://www.macroinnovation.com/images/mgmnt.pdf>. Acesso em: 19 nov. 2006.

MCELROY, M. W. The new knowledge management: knowledge and innovation. Disponível em: < http://www.macroinnovation.com/images/mgmnt.pdf > . Acesso em: 15 dez. 2004

NÚÑ̃EZ PAULA, I. Enfoque teórico-metodológico para la determinación de las necesidades que deben atender los sistemas de información en las organizaciones o comunidades. 2002.99 p. Tese (Doutorado)- Universidad de la Habana. Facultad de Comunicación, 2002.

PONJUÁN DANTE, G. Aplicaciones de la gestión de información en las organizaciones: el profesional de la información y su dominio de las técnicas y herramientas de la gestión. 2000. 119 p. Tese (Doutorado)- Universidad de la Habana. Facultad de Comunicación, 2000.

SILVA, E. Orozco. El lugar de la inteligencia empresarial en el entorno conceptual de la gestión del conocimiento: evolución en Cuba. El profesional de la Información, v. 10, n. 7-8, p. 14-22, 2001. Disponível em: < khttp://elprofesionaldelainformacion. metapress.com/(zhxt0irwcx3kdb55cgpuaczb)/app/home/contribution.asp?referrer $=$ parent\&backto $=$ issue,3,12; journal,32,47; homemainpublications, $1,1>$. Acesso em: 16 nov. 2005. 